\title{
Evaluating Blood Glucose-6-Phosphate Dehydrogenase Activity with Oxidative Stress: A Study in Uncomplicated Type 2 Diabetes Mellitus Patients
}

\author{
Ruchika Priyadarsini ${ }^{1}$, Pramila K Mishra ${ }^{2}$, Nirupama Devi ${ }^{3}$, Rasmita K Padhy ${ }^{4}$
}

\begin{abstract}
Background: Diabetes mellitus (DM) is chronic hyperglycemia condition affecting multiple organs due to metabolic disorder. Insulin secretion, function, or both are affected for which one of the factors attributed is due to increased free radical activity. Nicotinamide adenine dinucleotide phosphate (NADPH) produced in HMP shunt pathway is regulated by the rate-limiting glucose-6-phosphate dehydrogenase (G6PD). When there is an imbalance between the production of reactive oxygen species and the antioxidant system that detoxifies, then it is called oxidative stress. This pathway is regulated by the reductant concentration of NADPH.

Aims and objectives: The current study was taken up to evaluate and correlate oxidative stress and insulin resistance with G6PD activity in type 2 DM (T2DM) patients.

Materials and methods: A total of 100 (76 males 24 females) T2DM patients with equal age- and sex-matched healthy controls were selected for the study. Glucose-6-phosphate dehydrogenase was measured by chemical method in semiauto analyzer. Total oxidative stress measured as ferrous oxidation in xylenol orange and total antioxidant capacity estimated as ferric-reducing ability of serum by spectrophotometer. Glucose was measured by glucose oxidase-peroxidase method in an autoanalyzer. SPSS Version 20 software was used for statistical analysis.

Results and observations: Increased serum G6PD levels were found in DM patients which significantly correlates with the increase of oxidative stress and high glucose levels ( $p$ value $<0.01$ ).

Conclusion: Estimation of blood G6PD activity may be used as a test to know the extent of oxidative status in DM patients for its implications in further clinical complications.

Keywords: Fasting plasma glucose, Ferrous oxidation in xylenol orange, Ferric-reducing ability of serum, Glucose-6-phosphate dehydrogenase, Nicotinamide adenine dinucleotide phosphate.

Indian Journal of Medical Biochemistry (2020): 10.5005/jp-journals-10054-0134
\end{abstract}

\section{INTRODUCTION}

According to World Health Organization (WHO), ${ }^{1}$ as of October 2013, 347 million people have diabetes mellitus (DM). The WHO projects that the seventh leading cause of death worldwide will be DM by the year 2030. The state of Odisha comprises 44 million people, and according to estimates, around 1.5 million people in Odisha are suffering from DM against the country's 62 million. ${ }^{2}$ Many diseases are associated with oxidative stress, and type 2 DM (T2DM) is one of them. The etiopathogenesis is due to increase in oxidative stress along with inadequate antioxidant defense systems as revealed by many studies. A significant role is being played by free radicals in the progression of this multifactorial disease. The imbalance between generation of oxidants and antioxidants results in oxidative stress. Glucose auto-oxidation, protein glycation, formation of advanced glycation end products, etc. are the different intricate mechanisms resulting in origin of free radicals. These are equally damaging toward macromolecules such as DNA, molecules of extracellular matrix, lipoproteins, etc. ${ }^{3-5}$

These free radicals induce cellular damage and $\beta$-cell dysfunction causing decrease in insulin secretion and signaling. ${ }^{6}$ Generation and detoxification of reactive oxygen species (ROS) maintains the redox balance in normal physiological state. Any alteration in this tightly controlled system leads to cell damage and death. Redox balance is affected and regulated by many diseases including diabetes. ${ }^{7-11}$

The chief source of nicotinamide adenine dinucleotide phosphate (NADPH) is the HMP shunt pathway, regulated by the
1,2Department of Biochemistry, Maharaja Krushna Chandra Gajapati Medical College and Hospital, Brahmapur, Odisha, India

${ }^{3}$ Department of Biochemistry, Santha Bhima Bhoi Medical College and Hospital, Balangir, Gandhrel, Odisha, India

${ }^{4}$ Department of Biochemistry, Saheed Laxman Naik Medical College and Hospital, Koraput, Odisha, India

Corresponding Author: Ruchika Priyadarsini, Department of Biochemistry, Maharaja Krushna Chandra Gajapati Medical College and Hospital, Brahmapur, Odisha, India, Phone: +91 9437109024, e-mail: priyadarsiniruchika@gmail.com

How to cite this article: Priyadarsini R, Mishra PK, Devi N, et al. Evaluating Blood Glucose-6-Phosphate Dehydrogenase Activity with Oxidative Stress: A Study in Uncomplicated Type 2 Diabetes Mellitus Patients. Indian J Med Biochem 2020;24(1):4-8.

Source of support: Nil

Conflict of interest: None

antioxidant enzyme glucose-6-phosphate dehydrogenase (G6PD). $\mathrm{NADPH}$ is required for synthesis of nitric oxide, glutathione recycling within the cells, cytochrome P450, and others. ${ }^{12,13}$ Activity of G6PD is affected by various signal transduction mechanisms such as transcription, posttranslational modifications, sorting, and transport that require interaction with other proteins. ${ }^{14,15}$

Pronounced changes in environment, food availability, and lifestyle have resulted in escalating the rates of diabetes.

(O) The Author(s). 2020 Open Access This article is distributed under the terms of the Creative Commons Attribution 4.0 International License (https://creativecommons. org/licenses/by-nc/4.0/), which permits unrestricted use, distribution, and non-commercial reproduction in any medium, provided you give appropriate credit to the original author(s) and the source, provide a link to the Creative Commons license, and indicate if changes were made. The Creative Commons Public Domain Dedication waiver (http://creativecommons.org/publicdomain/zero/1.0/) applies to the data made available in this article, unless otherwise stated. 
Chronic hyperglycemia and its oxidant derivatives play a major role in pathogenesis of DM which is demonstrated by several research studies. ${ }^{16,17}$ Activity of enzyme G6PD as an antioxidant system is important in preventing and postponing complications of DM. ${ }^{18,19}$

\section{Aims and Objectives}

- The current study was designed to evaluate G6PD activity in uncomplicated T2DM.

- Oxidant activity and antioxidant activity were correlated with levels of G6PD.

\section{Materials and Methods}

This is a hospital-based, case-control prospective study. It includes 100 diagnosed cases of T2DM and equally matched healthy controls during the period of December 2014 to December 2015. Patients with autoimmune disorders, smokers, hypertension, alcoholics, and tobacco users were excluded in this study. Ethical Committee approval was obtained to conduct the study. Written consents were obtained from the participants (patient and control groups).

A morning sample of venous blood $(5 \mathrm{~mL})$ was collected after overnight fast in a day by sterile disposable syringe under aseptic condition. Serum was used for analysis of fasting insulin (FI), ferrous oxidation in xylenol orange (FOX-2). Plasma was used for the analysis of ferric-reducing ability of serum (FRAP) and glucose. All the tests were done within 8 hours of collection and separation of serum. Serum total oxidant level was measured by FOX-2 assay ${ }^{20}$ and measured spectrophotometrically at $560-\mathrm{nm}$ wavelength. The results were expressed in $\mu \mathrm{mol} \mathrm{H}_{2} \mathrm{O}_{2} / \mathrm{L}$. The total antioxidant capacity was measured by $\mathrm{FRAP}^{20}$ and read spectrophotometrically at a wavelength of $593 \mathrm{~nm}$. The results were expressed in $\mu \mathrm{mol} /$ liter equivalent of $\mathrm{FeSO}_{4}$ solution. Insulin was assessed by commercial kit supplied by US Diagnostics Roche. It was estimated in Roche Cobase411 autoanalyzer by electrochemiluminescence method and glucose by glucose oxidase and peroxidase (GOD-POD) method by fully automated Toshiba TBA 120 FR procuring kit from AGAPPE diagnostics. G6PD was estimated in vitro by kinetic method in blood at $340 \mathrm{~nm}$ by procuring kit from Far Diagnostics.

Statistical analysis of the data was done using independent Students $t$ test. Correlations were calculated by Karl Pearson's coefficient.

\section{Results}

In our study, we have seen that DM is more common in the middleaged group, with the mean age of patients being 52 years, more prevalent in males, and $79 \%$ of cases belonging to the age group of 40-60 years (Table 1 and Fig. 1).

The physical attributes such as age, body mass index (BMI), waist circumference, and blood pressure (BP: systolic and diastolic) were

Table 1: Age and sex distribution of cases in type 2 diabetes mellitus

\begin{tabular}{lccc}
\hline Age (years) & Males & Females & Total \\
\hline $30-40$ & 3 & 4 & 7 \\
$41-50$ & 25 & 11 & 36 \\
$51-60$ & 33 & 9 & 42 \\
$61-70$ & 13 & 2 & 15 \\
\hline
\end{tabular}

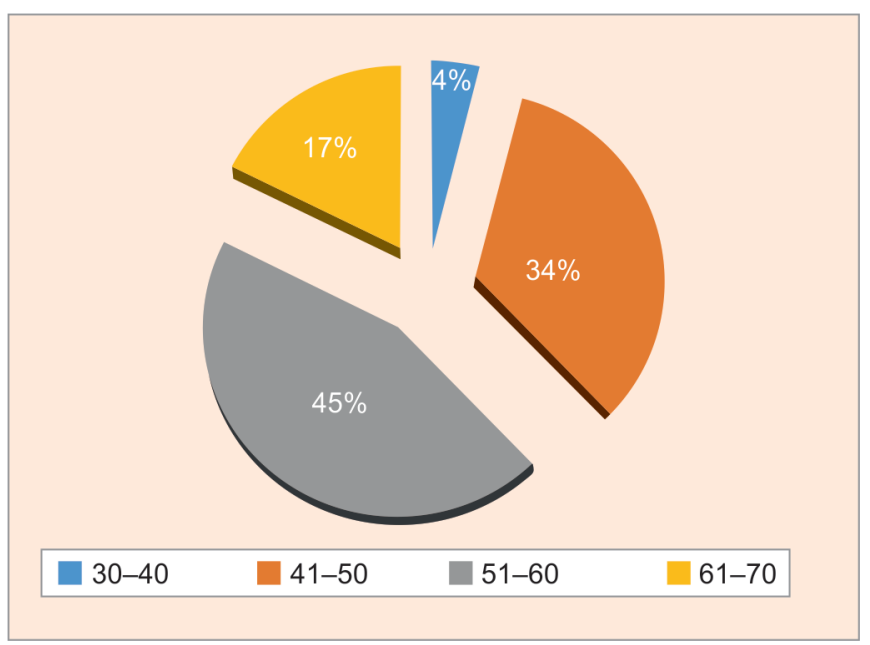

Fig. 1: Pie chart showing age distribution of cases in type 2 diabetes mellitus

Table 2: Physical attributes of diabetics and controls

\begin{tabular}{lccl}
\hline Parameters & $\begin{array}{l}\text { Diabetics } \\
(n=100)\end{array}$ & $\begin{array}{l}\text { Controls } \\
(n=100)\end{array}$ & p value \\
\hline Age (years) & $52.6 \pm 8.5$ & $51.5 \pm 10.2$ & 0.416 \\
BMI $\left(\mathrm{kg} / \mathrm{m}^{2}\right)$ & $28.2 \pm 4.9$ & $28.5 \pm 4.0$ & 0.690 \\
Waist circumference $(\mathrm{cm})$ & $99.7 \pm 9.93$ & $100.2 \pm 10.5$ & 0.295 \\
SBP $(\mathrm{mm} \mathrm{Hg})$ & $132.3 \pm 16.21$ & $131.2 \pm 31.6$ & 0.862 \\
DBP $(\mathrm{mm} \mathrm{Hg})$ & $81.6 \pm 10.8$ & $82.1 \pm 10.1$ & 0.295 \\
\hline
\end{tabular}

$\mathrm{BMI}$, body mass index; SBP, systolic blood pressure; DBP, diastolic blood pressure. Data is represented as mean \pm SD. $p$ value $<0.05$ is considered significant

elevated but not statistically significant among the study groups ( $p$ value $>0.05$ ) (Table 2 ).

The FOX-2, FI, and fasting plasma glucose (FPG) levels significantly raised in DM, showing increased oxidative stress in diabetic patients. Ferric-reducing ability of serum was significantly less in patients showing decreased antioxidant levels.

G6PD is an intracellular enzyme and barely shows any serum activity. ${ }^{21}$ But in our study, we found that serum activity is increased in diabetic patients $(1.92 \pm 0.55)$ when compared to healthy individuals $(0.09 \pm 0.06)$ as shown in Table 3 and Figure 2 .

The correlation between activity of G6PD and oxidative stress in diabetic individuals is shown in Figure 3. Thus, the level of G6PD in the plasma increases with the increase in oxidative stress.

On correlating G6PD with FPG, it was observed that in diabetic patients G6PD is positively and significantly correlated with FPG which shows that G6PD activity increases with hyperglycemia in diabetic cases Table 4 and Figure 4.

\section{Discussion}

Diabetes mellitus has become a global disaster placing considerable burden on healthcare services. Increased lifespan, population explosion, unplanned urbanization, low education level, increased external debt, and reduction in national healthcare expenditure are the reasons behind the "epidemiological transition" of this 
Table 3: Comparision of biochemical parameters of healthy control subjects and diabetic patients

\begin{tabular}{|c|c|c|c|c|c|}
\hline S. no & Parameters & Case $($ mean $\pm S D)$ & Control (mean $\pm S D)$ & pvalue & $t$ \\
\hline 1 & G6PD (mU/mL) & $1.92 \pm 0.55$ & $0.09 \pm 0.06$ & 0.00 & 32.59 \\
\hline 2 & FOX2 (equivalent of $\left.\mathrm{H}_{2} \mathrm{O}_{2}\right)(\mu \mathrm{mol} / \mathrm{L})$ & $6.51 \pm 1.45$ & $4.02 \pm 1.04$ & 0.00 & 13.91 \\
\hline 3 & FRAP (equivalent of ferrous sulfate) ( $\mu \mathrm{mol} / \mathrm{L}$ ) & $307.22 \pm 78.80$ & $643.01 \pm 49.22$ & 0.00 & -36.14 \\
\hline 4 & Fasting insulin $(\mu \mathrm{lU} / \mathrm{mL})$ & $16.35 \pm 7.72$ & $6.29 \pm 1.15$ & 0.00 & 12.89 \\
\hline 5 & $\mathrm{FPG}(\mathrm{mg} / \mathrm{dL})$ & $146.64 \pm 25.55$ & $96.39 \pm 10.29$ & 0.00 & 18.23 \\
\hline
\end{tabular}

FOX-2, ferrous oxidation in xylenol orange-2; FRAP, ferric-reducing ability of serum; G6PD, glucose-6-phosphate dehydrogenase. $p$ value $<0.05$ is considered significant.

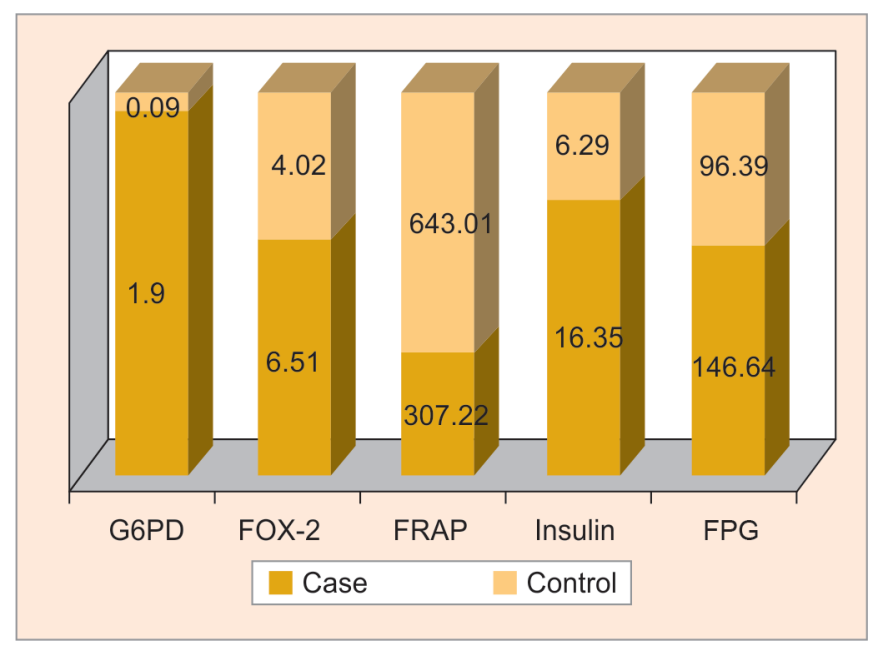

Fig. 2: Mean difference in biochemical parameters in cases and controls

disease. ${ }^{22}$ Studies shows that at a lower BMI in men has a higher tendency for visceral fat deposition and are more susceptible to insulin resistance leading to T2DM. ${ }^{23}$

A marker of oxidative stress damage is lipid hydroperoxides which is measured by FOX-2 assay method which was found to be elevated in diabetic patients. The pro-oxidant and antioxidant equilibrium in diabetic patients was estimated by measuring plasma lipid hydroperoxide levels. The total antioxidant levels were found significantly lower in plasma of DM patients when compared to normal healthy individuals. From a complex biological fluid system, it is difficult to isolate, identify, and estimate individual antioxidants. Due to chemical diversity, the collective action of all the natural antioxidants when assessed is potentially more useful toward an individual's health.

The increase in plasma lipid hydroperoxides levels and decrease in the antioxidant activity in T2DM patients indicate oxidative stress. The study carried out by Patel et al. ${ }^{24}$ provides direct evidence of free radical-induced injury associated with this disease. Insulin resistance, a risk factor for T2DM, develops when ROS and RNS have got downregulation effect on insulin-signaling receptor pathways.

NADPH produced from NADP via G6PD while oxidizing glucose6-phosphate is used for cell survival and helps in maintaining redox balance. It is exceptionally conserved in greater part of the mammalian species. Glucose-6-phosphate is a substrate for G6PD in HMP shunt and also for glycolytic pathway.

A slightly raised G6PD activity in patients with T2DM was observed by Joshi et al. ${ }^{25}$
Table 4: Correlation coefficients of different variables ( $R^{2}$ value)

\begin{tabular}{llll}
\hline Parameters & FOX-2 & FRAP & G6PD \\
\hline FPG & 0.585 & -0.709 & 0.735 \\
Fasting insulin & 0.404 & -0.643 & 0.659 \\
\hline
\end{tabular}

$p$ value $<0.001$

FOX-2, ferrous oxidation in xylenol orange-2; FRAP, ferric-reducing ability of serum; G6PD, glucose-6-phosphate dehydrogenase

The availability of $\mathrm{NAD}^{+}$and feedback inhibition by ATP decides the activity of HMP shunt pathway, as $10 \%$ of glucose is utilized by this pathway under normal physiological conditions. Oxidative stress causes increased flux of glucose through this pathway. As the ratio between NADPH-NADP decreases, G6PD activity increases to provide more NADPH. When cells are exposed to various external stimuli, the level of NADPH, the cells principal reductant, decreases. Activity of G6PD is triggered following diminished the level of NADPH. ${ }^{26}$ Hence, G6PD activity may increase in serum due to reduced availability of substrate glucose-6-phosphate in DM patients.

Superoxide dismutase, catalase, thioredoxin, and the glutathione system are some important antioxidant systems in our body. But until now, the antioxidant property of G6PD has not been properly evaluated. The functioning of entire antioxidant system depends on NADPH. ${ }^{12,13}$

Research done by Zhang et al. ${ }^{27}$ as well as others have indicated that chronic hyperglycemia impairs the activity of G6PD by decreasing NADPH production in vascular endothelial cells. They carried on their research work further and for the first time found that by increasing G6PD activity, there is a remarkable improvement in the level of redox enzymes and redox status. Henceforth, the change in redox status leads to decrease in cell death and enhanced cell growth in endothelial cells. A signaling molecule gets modified which influences the activities of other antioxidant enzymes due to overexpression of G6PD. This modified redox status affects the activity of ROS-scavenging antioxidant enzymes in the form of posttranslational modification. ${ }^{27}$

\section{Conclusion}

The present study showed an increase in G6PD activity in DM patients which was positively correlated with plasma hydroperoxide level measured as FOX-2. The plasma antioxidant levels measured by FRAP was found to be reduced in DM patients. Despite the fact that these enzymatic changes are comparatively very small, they are physiologically significant. Further studies 


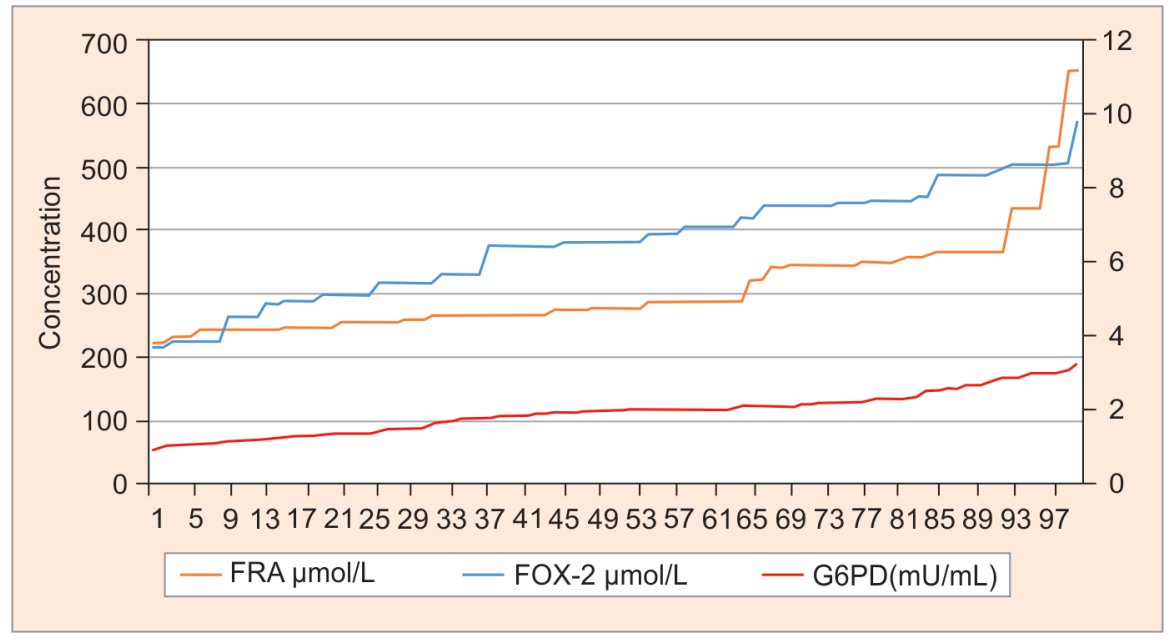

Fig. 3: Correlation of glucose-6-phosphate dehydrogenase activity with oxidative stress in type 2 diabetes mellitus cases

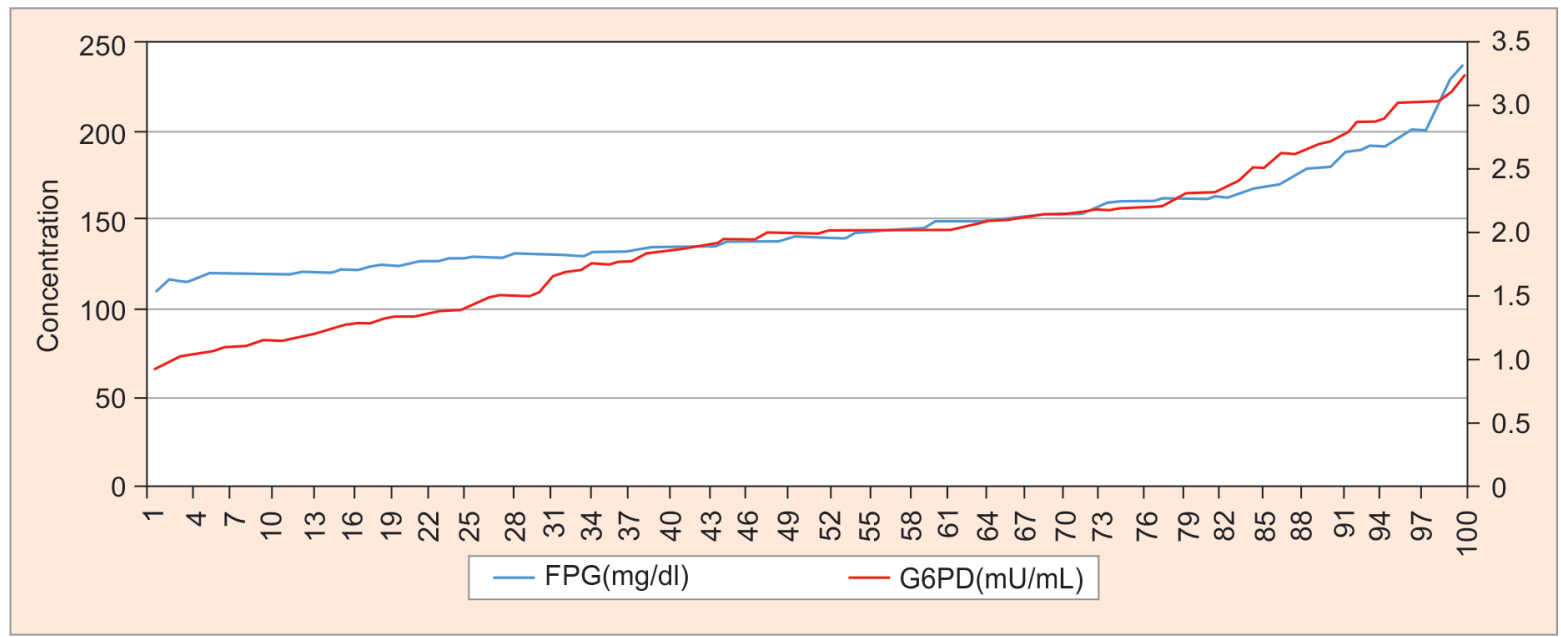

Fig. 4: Correlation of glucose-6-phosphate dehydrogenase activity with fasting plasma glucose in type 2 diabetes mellitus cases

in the larger group of patients is required for identification of the important role of G6PD activity. This knowledge will give us a clear understanding about the oxidative stress-attributed complications in T2DM.

\section{References}

1. Wild S, Roglic G, Green A, et al. Global prevalence of diabetes estimate for the year 2000 and projection for 2030. Diabetes Care 2004;27(5):1047-1053. DOI: 10.2337/diacare.27.5.1047.

2. Agrawal R, Rath B, Saha K, et al. Drug utilization pattern of antidiabetic agents in a tertiary care hospital of western Odisha, India. Int J Basic Clin Pharmacol 2016;5(5):2222-2226. DOI: 10.18203/2319-2003. ijbcp20163265.

3. Brownlee M. Biochemistry and molecular cell biology of diabetic complications. Nature 2001;414(6865):813-820. DOI: 10.1038/414813a.

4. Buse MG. Hexosamines, insulin resistance, and the complications of diabetes: current status. Am J Physiol Endocrinol Metab 2006;290(1):1-8. DOI: 10.1152/ajpendo.00329.2005.

5. Brownlee M. The pathobiology of diabetic complications: a unifying mechanism. Diabetes 2005;54(6):1615-1625. DOI: 10.2337/ diabetes.54.6.1615.

6. Mousa SA. Oxidative stress in diabetes mellitus. Romanian J Biophys 2008;18(3):225-236.
7. Fatokun AA, StoneTW,Smith RA. Oxidative stress in neurodegeneration and available means of protection. Front Biosci 2008;13(13): 3288-3311. DOI: 10.2741/2926.

8. Forbes JM, Coughlan MT, Cooper ME. Oxidative stress as a major culprit in kidney disease in diabetes. Diabetes 2008;57(6):1446-1454. DOI: $10.2337 / \mathrm{db} 08-0057$.

9. Lenzen S. Oxidative stress the vulnerable beta cell. Biochem Soc Trans 2008;36(Pt 3):343-347. DOI: 10.1042/BST0360343.

10. Lubos $E$, Handy DE, Loscalzo J. Role of oxidative stress \& nitric oxide in atherosclerosis. Front Biosci 2008;13(13):5323-5344. DOI: $10.2741 / 3084$.

11. Thomas SR, Witting PK, Drummond GR. Redox control of endothelial function \& dysfunction: molecular mechanism \& therapeutic opportunities. Antioxidant Redox Signals 2008;10(10):1713-1765. DOI: 10.1089/ars.2008.2027.

12. Frederick WM, Bosch $\mathrm{KS}$, Jong DJ, et al. Post translational regulation of glucose-6-phosphate dehydrogenase activity in (pre) neoplasti lesions in rat liver. J Histochem Cytochem 2003;51(1):105-112. DOI: 10.1177/002215540305100112.

13. Stanton RC. Glucose-6- phosphate dehydrogenase, NADPH \& cell survival. IUBMB Life 2012;64(5):362-369. DOI: 10.1002/iub. 1017. 
14. Brauwald E, Fauci AS, Longo DL, et al. Diabetes Mellitus. Harrison's Principle of Internal Medicine. 17th ed., (2), Newyork: MC Graw Hill; 2008. pp. 2275-2304.

15. Alberti KGMM, Zimmet P, Shaw J. International diabetes federation: a consensus on type 2 diabetes prevention. Diabet Med 2007;24(5): 451-463. DOI: 10.1111/j.1464-5491.2007.02157.x.

16. West IC. Radicles \& oxidative stress in diabetes. Diabet Med 2000;17(3):171-180. DOI: 10.1046/j.1464-5491.2000.00259.x.

17. Ramakrishna $V$, Jailkhani R. Evaluation of oxidative stress in insulin dependent DM (IIDM) patients. Diagn Pathol 2007;2(1):22. DOI: 10.1186/1746-1596-2-22.

18. Wan GH, Tsai SC, Chiu DT. Decreased blood activity of G6PD associated with increased risk of diabetes mellitus. Endocrine 2002;19(2):191-195. DOI: 10.1385/ENDO:19:2:191.

19. Zhang Z, Apse K, Pang J. High glucose inhibits G6PD via CAMP in aortic endothelial cells. J Biol Chem 2002;42(7):275-400.

20. Katalinic V, Salamunic I, Pazanin S, et al. The antioxidant power and lipid peroxidation products in the sera of apparently healthy adult males. Coll Antrpol 2007;1(1):165-171.

21. Varley's Practical Clinical Biochemistry. 6th ed, London: Heinemann Professional Publishing Ltd.
22. Mohan V, Mathur $P$, Deepa $R$, et al. Urban rural differences in prevalence of self reported diabetes in India-the WHO-ICMR Indian NCD risk factor surveillance. Diabetes Res Clin Pract 2008;80(1): 159-168. DOI: 10.1016/j.diabres.2007.11.018.

23. Sarwar N, Gao P, Seshasai SR, et al. Diabetes mellitus, fasting blood glucose concentration, and risk of vascular disease: a collaborative meta-analysis of 102 prospective studies. Lancet 2010;375(9733):2215-2222. DOI: 10.1016/S0140-6736(10)60484-9.

24. Patel V, Kanani D, Chauhan K, et al. Assessment of oxidative stress in type 2 diabetes mellitus by measurement of plasma lipid hydroperoxides and total antioxidant capacity. Int J Adv Res 2015;3(5):1078-1084.

25. Joshi SR, Joshi, Patel, et al. High prevalence of G6PD deficiency in Vataliya Prajapati community in Western India. Haematology 2001;31(1):57-60. DOI: 10.1163/15685590151092715.

26. European Medical Alliance $>>$ Haemolytic Anaemia. Defence against oxidative stress the production of reducing power. Last Updated on Wed, 08 Apr 2015. http://www.europeanmedical.info.

27. Zhang Z, Yang Z, Zhu B, et al. Increasing glucose 6-phosphate dehydrogenase activity restores redox balance in vascular endothelial cells exposed to high glucose. PLoS ONE 2012;7(11):e49128. DOI: 10.1371/journal.pone.0049128. 